\section{At What Age Was Systemic Lupus Erythematosus Diagnosed in This Cohort?}

\section{To the Editor:}

I read with great interest the paper on mortality and functionality after stroke in patients with systemic lupus erythematosus (SLE), by Rossides, $e t a l^{1}$, which fills important knowledge gaps in this domain.

However, I was surprised that the median age at stroke onset of the patients with SLE was 68 years for ischemic stroke and 65 for hemorrhagic stroke, while the mean time since SLE diagnosis was 2.5 years for ischemic stroke and 3.3 years for hemorrhagic stroke. This could have meant that the age at which SLE was diagnosed surpassed 60 years in this cohort, which is unusual for this disease.

Of course, it could have been a bit misleading that the time since SLE diagnosis is presented as mean, while it should have been summarized as median: when the SD is several times larger than the mean (15.5 for ischemic stroke and $\mathbf{1 7 . 6}$ for hemorrhagic stroke), the population is clearly not normally distributed, and median with interquartile range is more suitable.

CRISTIAN BĂICUŞ, MD, PhD, Carol Davila University of Medicine and Pharmacy, and Colentina University Hospital, Department of Internal Medicine, Bucharest, Romania. Address correspondence to Dr. C. Băicuş, Colentina Clinical Hospital, Department of Internal Medicine, Sos Stefan cel Mare 19-21, sector 2, Bucharest 020125, Romania.

E-mail: cbaicus@gmail.com

\section{REFERENCE}

1. Rossides M, Simard JF, Svenungsson E, von Euler M, Arkema EV. Mortality and functionality after stroke in patients with systemic lupus erythematosus. J Rheumatol 2017;44:1590-6.

J Rheumatol 2018;45:7; doi:10.3899/jrheum.171257 